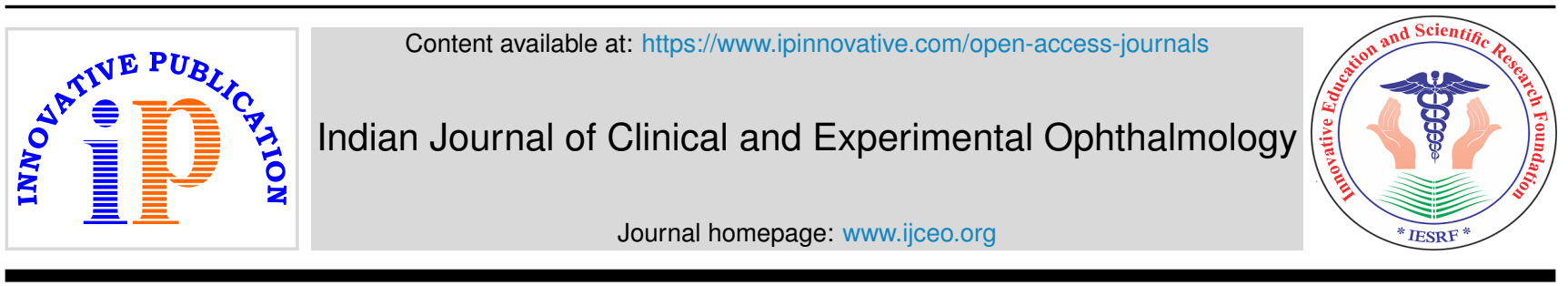

Original Research Article

\title{
Anatomical and functional significance of ectopic inner foveal layer (EIFL) in epiretinal membrane (ERM)
}

\author{
Sunil Ganekal ${ }^{1, *}$, Varun Ganekal ${ }^{1}$ \\ ${ }^{1}$ Dept. of Ophthalmology, Davangere Netralaya, Davangere, Karnataka, India
}

\section{A R T I C L E I N F O}

\section{Article history:}

Received 21-03-2021

Accepted 15-04-2021

Available online 30-06-2021

\section{Keywords:}

Epiretinal membrane

Ectopic inner foveal layer

\begin{abstract}
A B S T R A C T
Background/Objectives: To compare the visual acuity outcomes after surgical treatment of epiretinal membranes (ERM) in cases with and without ectopic inner foveal layers (EIFL).

Methods: A total of 100 eyes of 98 patients with idiopathic ERM were retrospectively staged according to the newer OCT classification and divided into two groups on the basis of presence or absence of EIFL. The two groups were evaluated for various parameters including visual acuity, thickness of the EIFL, reappearance of the foveal contour, outer retinal changes and complications before and after ERM peeling. Correlation analysis was done using chi square test and p-values were obtained for corresponding variables. Results: Of 100 eyes with ERM, 20\% had stage 1,29\% had stage 2, 42\% had stage3 \& 9\% had stage 4 disease. VA was worse in eyes with EIFL as compared those without it. Correlation of VA with EIFL thickness however was not significant. Surgical removal of ERM didn't alter EIFL frequency though the thickness decreased. New EIFL appeared in $4 \%$ eyes and 35\% demonstrated thickened fovea after surgery. Anatomical reappearance of foveal contour was poorer in eyes with EIFL. Outer retinal changes were more common in non EIFL eyes. Lamellar holes and nontractional cysts were more common in EIFL eyes.

Conclusion: EIFL is a significant prognostic tool to predict the visual outcome of surgery in cases of Epiretinal membrane. EIFL eyes did not regain foveal contour, had less outer retinal damage but were associated with poorer visual outcomes.

(c) This is an open access article distributed under the terms of the Creative Commons Attribution License (https://creativecommons.org/licenses/by/4.0/) which permits unrestricted use, distribution, and reproduction in any medium, provided the original author and source are credited.
\end{abstract}

\section{Introduction}

Epiretinal membranes (ERM) also known as cellophane maculopathy, macular pucker or Preretinal macular fibrosis depending upon the degree of severity. ERMs form over the retina due to fibrocellular proliferation at the vitreoretinal interface. ERMs are commonly idiopathic but may be associated with retinal vascular disease, intraocular surgery or retinal detachment. ${ }^{1}$

Various theories have been put through trying to provide an understanding regarding the pathophysiology of ERMs. A classically accepted theory is that cells from the retina migrate over the surface of the ILM through the microbreaks in the ILM caused by Posterior Vitreous Detachment

\footnotetext{
* Corresponding author.

E-mail address: drgsunil@yahoo.com (S. Ganekal).
}

(PVD). ${ }^{2}$ Another theory suggests that ERMs are formed by the proliferation of the remnants of the cortical vitreous left on the premacular region after an anomalous PVD has occurred. ${ }^{3}$ The identification of exact lineage of the cells which proliferate to form ERMs is difficult because of the ability of these cells to transdifferentiate. ${ }^{4}$ There is evidence that these cells are derivatives of Muller cells or astrocytes. ${ }^{5}$ Other cells which have been identified in ERMs are hyalocytes, cells of Retinal Pigment Epithelium (RPE), fibroblasts, myofibroblasts. The myofibroblasts are thought to mediate the deposition of collagen, and formation of contractile proteins thus mediating the contraction of ERMs. ${ }^{6}$ The extracellular matrix of idiopathic ERM is likely to be composed of type II collagen. Ectopic Inner foveal layers are defined as the presence of a continuous hyporeflective or hyperreflective band, extending from the 
inner nuclear layer and the inner plexiform layer across the foveal region and visible in all OCT scans centered in the fovea.

On the basis of their appearance on SD-OCT, the ERMs have been classified into four stages (FIG 1). Stage 1 is defined as mild ERM with negligible morphologic or anatomical disruption of underlying retina. Stage 2 ERM is defined as progressive retinal distortion resulting in loss of foveal depression and characteristic stretching of the outer nulear layer towards the ILM. Stage 3 ERMs are those with Ectopic Inner Foveal Layers (EIFL) anomalously crossing the center of the fovea and clearly identifiable inner retinal layers. Stage 4 ERMs were those with EIFL and marked anatomical disruption of the macula resulting in undifferentiable inner retinal layers.

The role of the inner retinal layers in visual acuity loss has been studied, as these inner layers may be the primary affected site of ERM-associated mechanical stress. Ganglion cell-innerplexiform layer complex thickening was significantly associated with visual acuity reduction in various reports, while some authors have proposed the inner retinal irregularity index as a valid tool for postoperative visual prognosis in eyes with ERMs. In present study we compared the functional and anatomical outcomes after surgical treatment of epiretinal membranes (ERM) in cases with and without ectopic inner foveal layers.

\section{Materials and Methods}

This was a cohort analysis of a total of 100 eyes of 98 patients diagnosed with idiopathic ERMs and treated at our institute. This study was approved by the Institutional review board. Cases were identified by Medical records search for the diagnosis of ERM or macular pucker or cellophane maculopathy.

The inclusion criteria were the cases of unilateral or bilateral idiopathic ERMs with a minimum of 12 months follow up. Cases with a minimum post-operative follow up of 12 months were taken if they underwent surgical treatment for ERM. Exclusion criteria were any history of previous intraocular surgery with an exception of uncomplicated phacoemulsification, history of retinal detachment, presence of ARMD, CNVM due to any cause, proliferative diabetic retinopathy, Diabetic macular edema, retinal vein occlusions, glaucoma and presence of any other potential cause of vision loss other than ERM.

OCT images obtained using hiedelberg engineering spectralis- Spectral domain OCT were reviewed using hiedelberg eye explorer software. The eyes which underwent surgical treatment were divided into two groups on the basis of newer OCT based classification of ERMs. ${ }^{7}$ Eyes without EIFL i.e. those with Stage 1 and Stage 2 ERMs were classified as one group and called as non-EIFL group while the eyes having EIFL i.e. Stage 3 and Stage 4 ERMs were classified as other group called
EIFL group. The groups were analyzed for BCVA, Central foveal thickness (CFT), EIFL thickness, and changes in foveal contour, presence of intraretinal cysts, lamellar / full thickness defects in retinal layers and outer retinal changes which included defects in external limiting membrane, ellipsoid zone and interdigitation zone. Corresponding parameters were noted for both preoperative visit and a visit after post operative follow up of 12 months.

The BCVA which was measured using a Snellen's chart was converted to $\log$ MAR visual acuity. A single averaged high-definition horizontal line scan passing through the fovea taken on each of the two visits using Spectralis SD-OCT was analyzed for each eye for all quantitative measurements. The "Caliper" tool provided in the Hiedelberg Eye Explorer software was used to measure the thickness of ectopic inner foveal layers. A volume scan of central $30^{\circ} \times 25^{\circ}$ was taken as 61 horizontal line scans, each $128 \mu \mathrm{m}$ apart. Automated Mean Central Foveal thickness was obtained using "thickness map" tab in the software. Considering the upper limit of outer nuclear layer as their inferior border, the thickness of ectopic inner foveal layers was measured upto the inner border of the internal limiting membrane using "caliper" tool (Figure 2).

All patients who met the study criteria were included in the study during the recruitment period. Descriptive statistics are expressed as mean \pm standard deviation or median (minimum-maximum) for continuous variables and as frequency and percentage for categorical variables. The normal distribution of the continuous variables was tested using the Kolmogorov-Smirnov test, and the homogeneity of variance was tested using the Levene test. The data show a non-normal distribution, and the variance between groups of ERM stages was not similar. Therefore, nonparametric tests were used for the analysis. The analysis of categorical data in cross tables was performed using the likelihood ratio test or the Fisher's exact test if any expected count was less than 5; otherwise, it was performed using the Pearson's Chi-square test. Analysis of the data was performed using IBM SPSS Statistics 17.0 software (IBM Corporation, Armonk, NY, USA). The results were considered statistically significant when $\mathrm{p}$ was less than 0.05 .

\section{Results}

Medical records of a total of 205 patients were identified through a manual search for the terms "ERM" or "Macular pucker" or "Cellophane maculopathy". One hundred and seven patients were excluded for the presence of single or multiple exclusion criteria. The remaining 98 patients who passed the exclusion criteria were enrolled into the study, of them $50(51.02 \%)$ were male and $48(48.97 \%)$ were females. Mean age of the patients was $65.49 \pm 18.66$ yrs. (range 23-88 years). Two of the study subjects had bilateral ERM. 


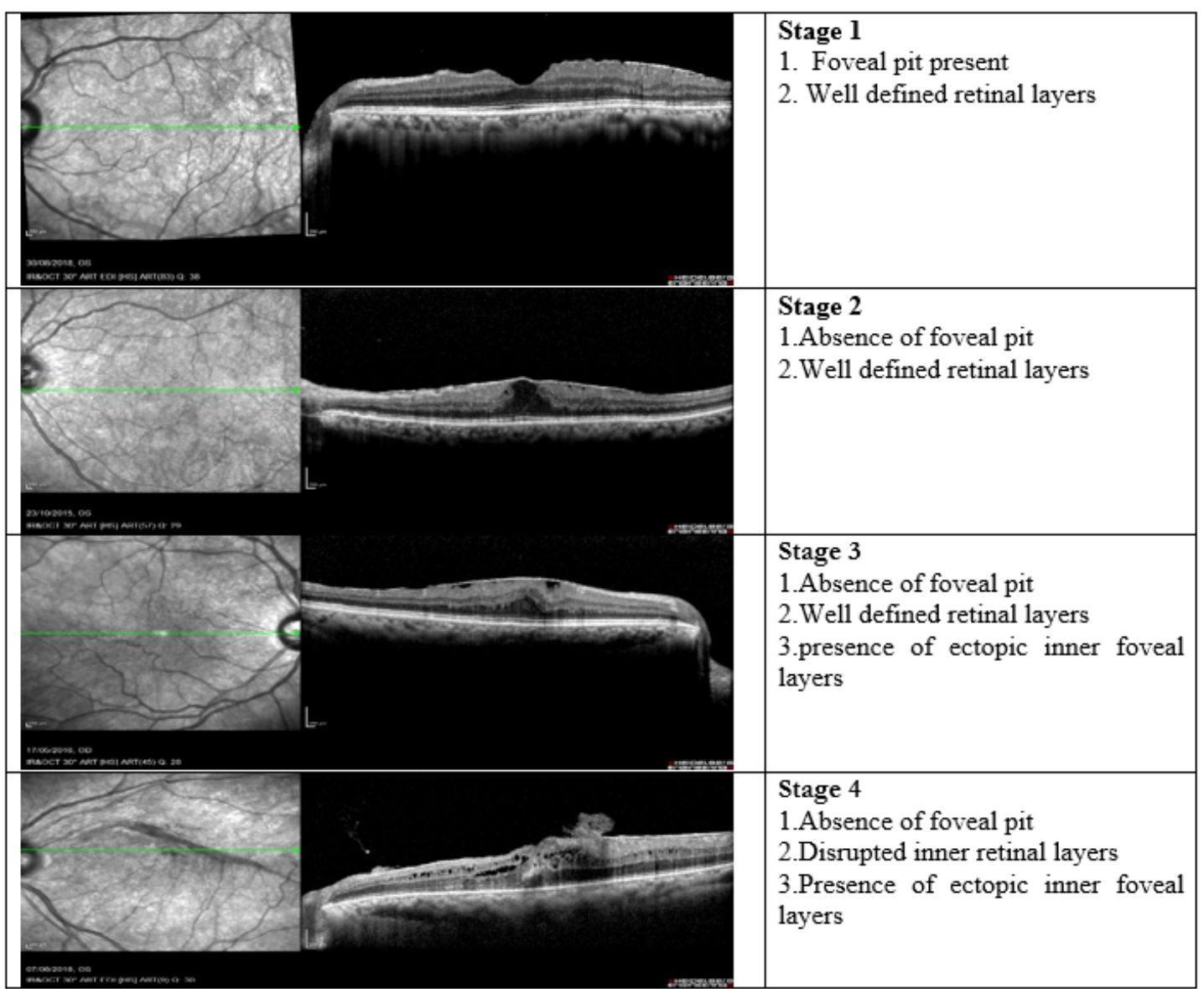

Fig. 1: OCT based staging of epiretinal membranes

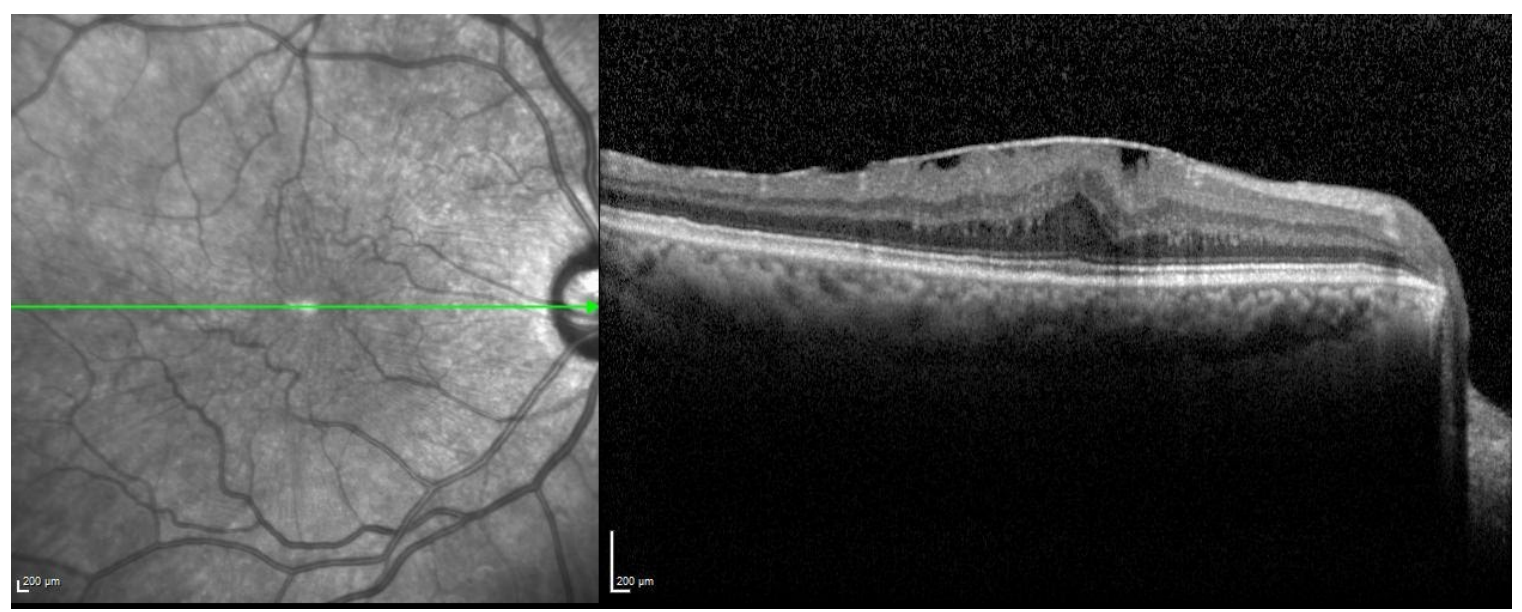

Fig. 2: Measurement of Ectopicinner fovel layer thickness- The upper limit of the outer nuclear layer is the inferior border of the ectopic inner foveal layers. The thickness of the ectopic inner foveal layers is measured by tracing a straight line from the upperlimit of the outer nuclear layer to the internal limiting membrane 

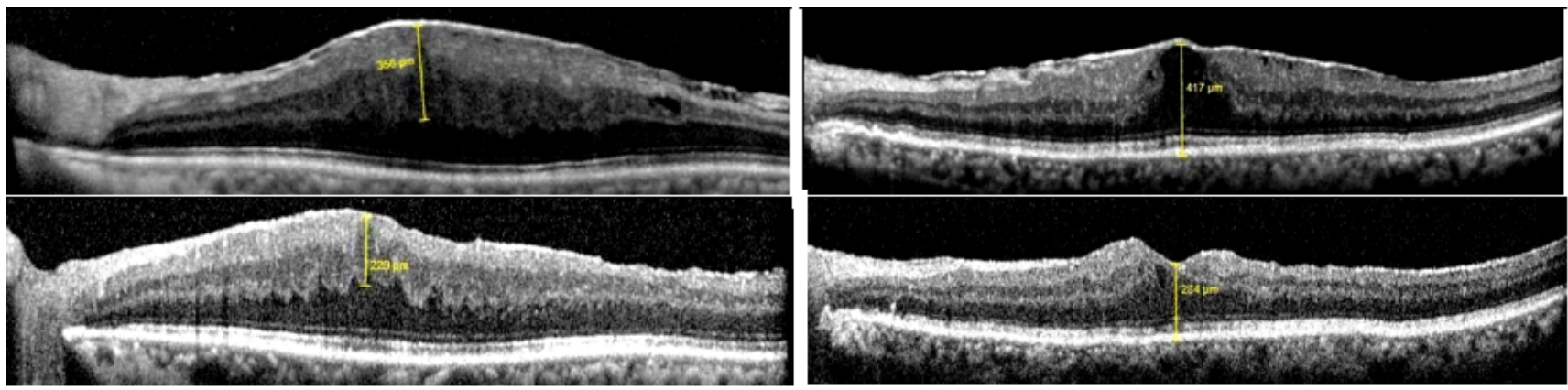

Fig. 3: (Left) OCT images of a case of ERM with Ectopic inner foveal layers: Thickness of EIFL is measured to show the reduction after surgery. (Right) OCT images of ERM without EIFL. Retinal thickness is measured to demonstrate the reduction in thickness, reappearance of foveal contour is also seen
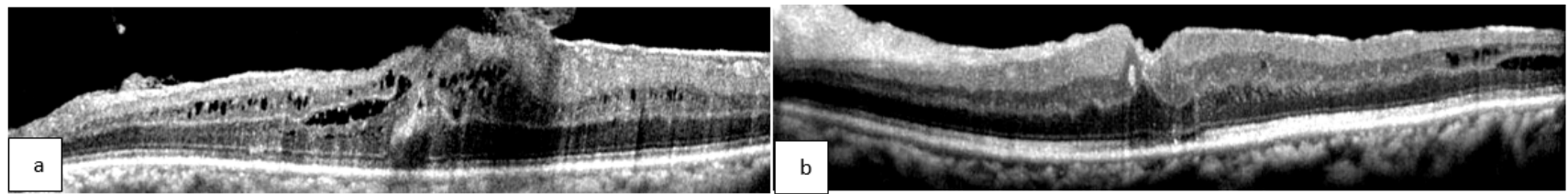

Fig. 4: a: OCT image of ERM with EIFL showing intraretinal cysts; b: OCT image of ERM without EIFL showing outer retinal changes

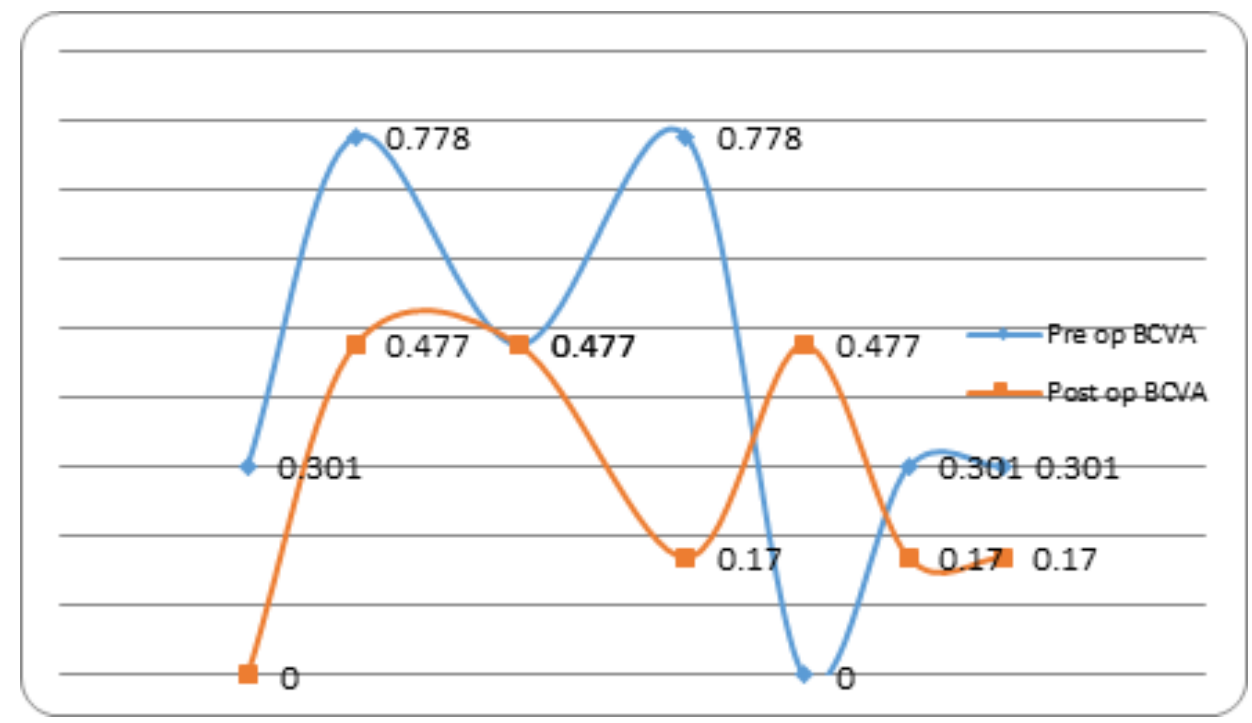

Fig. 5: Variation of BCVA (log MAR) with EIFL thickness

Twenty of the 100 study eyes had Stage 1 ERM, 29 had stage 2 ERM, 42 had stage 3 ERM and 9 had stage 4 ERM. The mean central foveal thickness of all the study eyes was $446.78 \pm 83.652 \mu \mathrm{m}$. Ectopic inner foveal layers were identified in 51 of 100 eyes under study with a mean EIFL thickness of $132.14 \pm 76.52 \mu \mathrm{m} .49$ out of 100 eyes had stage 1 or 2 ERM and were grouped as "non-EIFL" group while 51 out of 100 had stage 3 or 4 ERM and were grouped as the "EIFL" group. 8 eyes of the non EIFL group and 7 eyes of the EIFL group underwent total pars plana vitrectomy with ILM peeling.
The non-EIFL group had a mean age of $72.32 \pm 11.25$ years as compared to younger EIFL group with mean age $59.63 \pm 14.25$ years. The EIFL group had thicker foveae with mean foveal thickness of $529.14 \pm 48.07 \mu \mathrm{m}$ as compared to non-EIFL group with $505.12 \pm 82.03 \mu \mathrm{m}$. The mean preoperative BCVA of the non-EIFL group was better (0.31 $\pm 0.29 \log$ MAR, 20/41 snellen equivalent) as compared to the mean pre operative BCVA of the EIFL group (0.41 $\pm 0.28 \log$ MAR, 20/52 snellen equivalent). The mean thickness of EIFL was $178.85 \pm 86.65 \mu \mathrm{m}$. the correlation of BCVA with thickness of ectopic inner foveal layers 


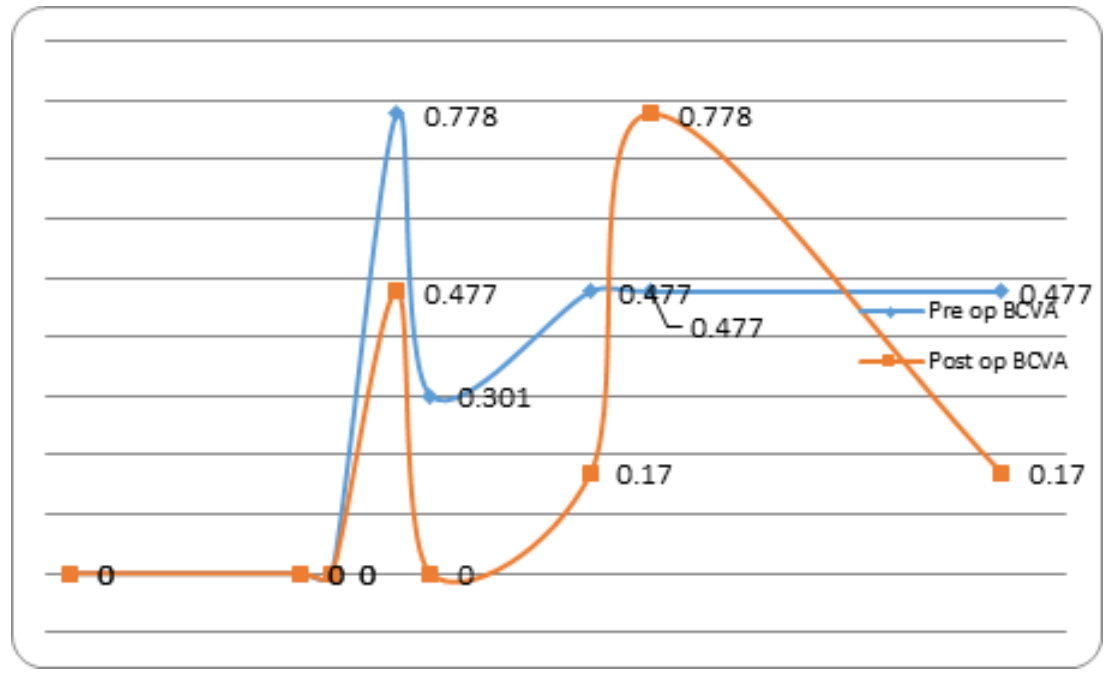

Fig. 6: Variation of BCVA (log MAR) with Central foveal thickness in non-EIFL eyes

was not statistically significant $(\mathrm{p}=0.062)$ Foveal contour reappeared in 3 out of 8 eyes in the non EIFL group while none of the operated eyes in EIFL group could regain the foveal contour after 12 months of surgery. The mean central foveal thickness of non-EIFL group reduced by $96 \mu \mathrm{m}$ post operatively and was evident as better post operative BCVA $(0.19 \pm 0.28 \log$ MAR, $20 / 31$ snellen equivalent). In the EIFL group the mean central foveal thickness reduced by only $53 \mu \mathrm{m}$ after surgery (Figure 3). Mean EIFL thickness $(142.47 \pm 42.07 \mu \mathrm{m})$ also came down in EIFL group but BCVA did not improve as much $(0.27 \pm 0.19 \log$ MAR, 20/37 snellen equivalent) after surgery. Intraretinal cysts were seen in 5 out of 8 eyes in EIFL group and none in the non-EIFL group. Outer retinal defects were more frequent (6 out of 7 ) in non-EIFL eyes as compared to EIFL eyes (5 out of 8). The central foveal thickness increased in two eyes of the EIFL group and 3 of the non EIFL group.

\section{Discussion}

The presence of EIFL before surgery has been significantly associated with poorer pre operative BCVA poorer gains in BCVA after surgery. ${ }^{7,8}$ However the correlation of EIFL with BCVA was not significant in our study. The BCVA improved in both groups after surgery. The EIFL eyes in general had poorer BCVA and also showed poorer gain in BCVA after surgery (Figures 5 and 6). Reduction in macular thickness after surgical treatment of ERM have been well reported in previous studies. ${ }^{9,10} \mathrm{We}$ found substantial reduction in the mean central foveal thickness in both groups after surgery. The ectopic inner foveal layers persisted post surgically in all the eyes but the thickness of EIFL reduced. This finding is similar to that of a previous study. ${ }^{8}$
The complications are caused due to the tractional stress induced by the ERMs. These complications involve all retinal layers and range from increased retinal thickness, formation of tractional intraretinal cysts, schisis and lamellar to full thickness retinal defects. Causes of visual disturbance may be metamorphopsia caused by macular distortion or edema. The combination of intraretinal gliosis and physical traction could result in formation of Ectopic inner foveal layers which may lead to loss of visual acuity by traction on inner retinal layers and obstructing the incident light from reaching the photoreceptors. The more frequent presence of Intra retinal cysts in EIFL group may be a consequence of damage to the retinal layers caused by the EIFL (Figure 5).The more frequent presence of outer retinal changes in the non-EIFL (Figure 6) group in our study suggests that the EIFL, during their formation, may bear a substantial part of tractional stress over the retinal layers saving the deeper layers which otherwise would have taken significant damage leading to photoreceptors loss.

\section{Conclusion}

Ectopic inner foveal layers are a significant prognostic tool to predict the outcome of surgery in Epiretinal membranes. As EIFL are formed in the latter 2 stages of ERMs, this may guide us as to when should a surgery be advised to expect the best possible post-operative visual out come. Once the EIFL have formed the visual prognosis gets worse.

\section{Source of Funding}

None.

\section{Conflict of Interest}

The authors declare that there is no conflict of interest. 


\section{References}

1. Schumann RG, Gandorfer A, Ziada J, Scheler R, Schaumberger MM, Wolf A. Hyalocytes in idiopathic epiretinal membranes: a correlative light and electron microscopic study. Graefes Arch Clin

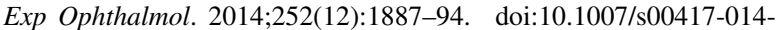
$284 I-X$

2. Foos RY. Vitreoretinal juncture - Simple epiretinal membranes. Albrecht Von Graefes Arch Klin Exp Ophthalmol . 1974;189(4):23150. 101:-10.1001/6+02384852.

3. Kampik A. Pathology of epiretinal membrane, idiopathic macular hole, and vitreomacular traction syndrome. Retina. 2012;32(Supplement 2):S194-9. do: 10.1097/ae.0b013e31825 hc20a

4. Vinores SA, Campochiaro PA, Mcgehee R, Orman W, Hackett SF, Hjelmeland LM. Ultrastructural and immunocytochemical changes in retinal pigment epithelium, retinal glia, and fibroblasts in vitreous culture. Invest Ophthalmol Vis Sci. 1990;31(12):2529-45.

5. Bringmann A, Wiedemann P. Involvement of Müller glial cells in epiretinal membrane formation. Graefes Arch Clin Exp Ophthalmol. 2009;247(7):865-83. 10i:101007/s004/7-000-1082-x

6. i Kohno R, Hata Y, Kawahara S, Kita T, Arita R, Mochizuki Y, et al. Possible contribution of hyalocytes to idiopathic epiretinal membrane formation and its contraction. Br J Ophthalmol. 2009;93(8):1020-6. do1:10.1136/bj0.2008.155069

7. Govetto A, Lalane RA, Sarraf D, Figueroa MS, Hubschman JP. Insights Into Epiretinal Membranes: Presence of Ectopic Inner Foveal Layers and a New Optical Coherence Tomography Staging Scheme. Am J Ophthalmol. 2017;175(3):99-113. do1:10.1016/].aj0.2016.12.006
8. Govetto A, Virgili G, Rodriguez FJ, Figueroa MS, Sarraf D, Hubschman JP. Functional and anatomical significance of the ectopic inner foveal layers in eyes with idiopathic epiretinal membranes: surgical results at 12 months. Retina. 2019;39(2):34757. 101:0.1097/1AE000000000001940.

9. Romano MR, Cennamo G, Schiemer S, Rossi C, Sparnelli F, Cennamo G. Deep and superficial OCT angiography changes after macular peeling: idiopathic vs diabetic epiretinal membranes. Graefes Arch Clin Exp Ophthalmol. 2017;255(4):681-9. 100101007/s004 7-016 B534-4.

10. Romano MR, Cennamo G, Cesarano I, Cardone D, Nicoletti G, Mastropasqua R, et al. Changes of Tangential Traction after Macular Peeling: Correlation between en-face Analysis and Macular Sensitivity. Curr Eye Res. 2017;42(5):780-8. 101:10.1080/027136832016. 231322.

\section{Author biography}

Sunil Ganekal, Medical Director

Varun Ganekal, Research Assistant

Cite this article: Ganekal S, Ganekal V. Anatomical and functiona significance of ectopic inner foveal layer (EIFL) in epiretinal membrane (ERM). Indian J Clin Exp Ophthalmol 2021;7(2):366-371. 